\title{
SUR LA THÉORIE SPECTRALE LOCALE DES SHIFTS À POIDS OPÉRATEURS
}

\author{
MOHAMED HOUIMDI ET HASSANE ZGUITTI
}

Reçu le 7 juin 2004 et en forme révisée le 7 mars 2005

Nous étudions les propriétés spectrales locales du shift unilateral à poids opérateurs. Nous donnons une condition nécessaire et suffisante pour que l'adjoint satisfasse la propriété de l'extension unique (SVEP). Une condition suffisante pour satisfaire la propriété de Dunford $(C)$ ainsi qu'une condition nécessaire pour satisfaire la condition de Bishop $(\beta)$ seront données. Enfin, nous montrons que le shift à poids opérateurs est décomposable si, et seulement si, il est quasinilpotent.

\section{Introduction}

Soient $H$ un espace de Hilbert séparable complexe de dimension infinie et $\mathscr{L}(H)$ l'algèbre des opérateurs linéaires bornés sur $H$. On désigne par $H^{(\infty)}$ l'espace des suites $\left(x_{n}\right)_{n \geq 0}$ définie par $H^{(\infty)}=\left\{\left(x_{n}\right)_{n \geq 0}: \forall n \geq 0, x_{n} \in H\right.$ et $\left.\sum_{n=0}^{\infty}\left\|x_{n}\right\|^{2}<\infty\right\}$. Muni du produit scalaire défini par $\langle x / y\rangle=\sum_{n=0}^{\infty}\left\langle x_{n}, y_{n}\right\rangle,\left(x=\left(x_{n}\right)_{n \geq 0}\right.$ et $\left.y=\left(y_{n}\right)_{n \geq 0} \in H^{(\infty)}\right), H^{(\infty)}$ est un espace de Hilbert. A chaque opérateur linéaire borné $T: H^{(\infty)} \rightarrow H^{(\infty)}$ est associé une matrice $\left(T_{n, m}\right)_{n, m \geq 0}$ à coefficients dans $\mathscr{L}(H)$.

Un opérateur $T: H^{(\infty)} \rightarrow H^{(\infty)}$ est dit un shift à poids opérateurs de poids $\left(T_{n}\right)_{n \geq 0}$, si les deux conditions suivantes sont satisfaites :

(i) $\sup _{n \geq 0}\left\|T_{n}\right\|<\infty$,

(ii) pour tout $x=\left(x_{n}\right)_{n \geq 0}$ de $H^{(\infty)}, T x=\left(0, T_{0} x_{0}, T_{1} x_{1}, \ldots, T_{n} x_{n}, \ldots\right)$.

La matrice d'un tel opérateur s'écrit sous la forme :

$$
T=\left(\begin{array}{ccccc}
0 & 0 & \cdots & 0 & \cdots \\
T_{0} & 0 & & & \\
0 & \ddots & \ddots & & \\
\vdots & 0 & T_{n} & 0 & \cdots \\
& & \ddots & \ddots & \ddots
\end{array}\right)
$$


3498 Sur la théorie spectrale locale des shifts à poids opérateurs

L'adjoint de $T$ est défini par

$$
T^{*} x=\left(T_{0}^{*} x_{1}, T_{1}^{*} x_{2}, \ldots, T_{n}^{*} x_{n+1}, \ldots\right) \quad \text { pour tout } x=\left(x_{n}\right)_{n \geq 0} \in H^{(\infty)} .
$$

On note par $H \hat{\otimes} H$ le complété du produit tensoriel hilbertien de $H$ par $H$. On a

(i) $\left(e_{n} \otimes e_{m}\right)_{n, m \geq 0}$ est une base orthonormale de $H \hat{\otimes} H$;

(ii) L'application $V$ de $H^{(\infty)}$ dans $H \hat{\otimes} H$ définie par $V\left(\left(x_{n}\right)_{n \geq 0}\right)=\sum_{n \geq 0}^{\infty} x_{n} \otimes e_{n}$ est unitaire.

Alors le shift à poids opérateurs $T$, de poids $\left(T_{n}\right)_{n \geq 0}$, peut être défini sur $H \hat{\otimes} H$ par

$$
T\left(e_{n} \otimes e_{m}\right)=T_{m} e_{n} \otimes e_{m+1} .
$$

Donc $T\left(e_{n} \otimes e_{m}\right)=T_{m} e_{n} \otimes S e_{m}$, où $S$ est le shift unilateral sans poids sur $H$.

Si $A \in \mathscr{L}(H)$ et $T_{n}=\alpha_{n} A$, pour tout $n \geq 0$ avec $\left(\alpha_{n}\right)_{n \geq 0}$ une suite bornée de nombres réels positifs, alors le shift à poids $T$, de poids $\left(T_{n}\right)_{n \geq 0}$, vérifie $T=A \otimes S_{\alpha}$.

Lorsqu'on identifie $H$ à $\mathbb{C}^{(\infty)}$, on retrouve la définition usuelle d'un shift unilateral à poids scalaires. Rappelons que $S_{\alpha}$ est un shift unilateral, de poids $\left(\alpha_{n}\right)_{n \geq 0}$, si

$$
S_{\alpha} e_{n}=\alpha_{n} e_{n+1}, \quad \forall n \geq 0,
$$

où $\left(e_{n}\right)_{n \geq 0}$ est une base orthonormale de $H$.

Dans le cas d'un shift à poids scalaires $S_{\alpha}$ de poids $\left(\alpha_{n}\right)_{n}$, nous utiliserons les notations adoptées par [15] : Soit $(\beta(n))_{n}$ la suite définie par

$$
\beta(0)=1, \quad \beta(n)=\alpha_{0} \cdots \alpha_{n-1} \quad \text { pour } n \geq 1 .
$$

Posons $r_{2}(T)=\liminf _{n \rightarrow \infty} \beta(n)^{1 / n}$.

Et si $S_{\alpha}$ est bilatéral (c'est-à-dire $n$ parcourt $\mathbb{Z}$ ) on pose $\beta(-n)=1 / \alpha_{-1} \cdots \alpha_{-n}$ pour $n \geq 1$.

Et soit aussi

$$
\begin{array}{cc}
r_{1}^{+}(T)=\lim _{n \rightarrow \infty}\left[\inf _{j \geq 0} \frac{\beta(n+j)}{\beta(j)}\right]^{1 / n}, & r^{+}(T)=\lim _{n \rightarrow \infty}\left[\sup _{j \geq 0} \frac{\beta(n+j)}{\beta(j)}\right]^{1 / n}, \\
r_{1}^{-}(T)=\lim _{n \rightarrow \infty}\left[\inf _{j<0} \frac{\beta(-n+j)}{\beta(j)}\right]^{1 / n}, & r^{-}(T)=\lim _{n \rightarrow \infty}\left[\sup _{j<0} \frac{\beta(-n+j)}{\beta(j)}\right]^{1 / n} .
\end{array}
$$

L'étude de la théorie spectrale locale des opérateurs shifts à poids scalaires a fait l'objet de plusieurs travaux, on cite par exemple les références $[4,13,14,16,17]$. Cependant, il apparaît qu'aucune étude satisfaisante des propriétés spectrales locales d'un shift à poids opérateurs n'est encore faite.

Dans ce papier, nous étudions les propriétés spectrales locales des shifts à poids opérateurs. Dans la Section 2 nous nous intéressons à la description du spectre et du spectre local. La première partie de la Section 3 sera consacrée à l'étude de la SVEP de l'adjoint; en plus nous donnons une condition nécessaire et suffisante pour avoir la propriété de l'extension unique. Dans la seconde partie, nous donnons une condition suffisante pour qu'un shift à poids opérateurs satisfasse la propriété de Dunford $(C)$. 
Nous montrons en particulier que, contrairement au cas du poids scalaire, le fait que $r_{S_{\alpha}}(x)=r\left(S_{\alpha}\right)$ pour tout $x \in H$ non nul, n'est plus une condition nécessaire pour qu'un shift à poids opérateurs satisfasse la propriété de Dunford $(C)$. Dans la dernière section nous complétons et nous généralisons les résultats figurant dans [13]. Nous établisserons des conditions nécessaires pour qu'un opérateur shift satisfasse la condition de Bishop $(\beta)$. Pour cela commençons par un rappel sur les notions de la théorie spectrale locale.

Soit $T$ un opérateur linéaire borné sur un espace de Banach $X$. On notera par $T^{*}$, $\sigma(T), \sigma_{a p}(T), \sigma_{p}(T)$ et $N(T)$ respectivement l'adjoint, le spectre, le spectre approximatif, le spectre ponctuel et le noyau de $T$. Soient $m(T)=\inf \{\|T x\|:\|x\|=1\}$ la borne inférieure de $T$ et $r_{1}(T)=\lim _{n \rightarrow \infty}\left(m\left(T^{n}\right)\right)^{1 / n}$. Soit $x \in X$, on note par $\sigma_{T}(x)$ le spectre local de $T$ en $x$. On rappelle que $\lambda \notin \sigma_{T}(x)$ s'il existe une fonction $f: V_{\lambda} \rightarrow X$ analytique sur un voisinage ouvert $V_{\lambda}$ de $\lambda$ telle que $(T-\mu) f(\mu)=x$, pour tout $\mu \in V_{\lambda}$. Et on a $\sigma_{s}(T)=\bigcup_{x \in X} \sigma_{T}(x)$, où $\sigma_{s}(T)=\{\lambda \in \mathbb{C}:(T-\lambda)(X) \neq X\}$ le spectre de surjectivité de $T$. On dit que $T$ satisfait la propriété de l'extension unique (notée brièvement la SVEP) s'il n'existe pas d'ouvert de $\mathbb{C}$ sur lequel l'équation $(T-\mu) f(\mu)=0$ admet une solution analytique non nulle; et dans ce cas on a $\sigma(T)=\sigma_{s}(T)$, [12]. Le rayon spectral local de $T$ en $x \in X$ non nul, noté $r_{T}(x)$, est défini par $r_{T}(x)=\limsup _{n \rightarrow \infty}\left\|T^{n} x\right\|^{1 / n}$. Si $T$ satisfait la SVEP, alors $r_{T}(x)=\sup \left\{|\lambda|: \lambda \in \sigma_{T}(x)\right\}$. On rappelle que $T$ satisfait la propriété de Dunford $(C)$ si le sous-espace spectral analytique $X_{T}(F)=\left\{x \in X: \sigma_{T}(x) \subseteq F\right\}$ est fermé pour toute partie $F$ fermée de $\mathbb{C}$ (voir [12]). Pour $U$ ouvert de $\mathbb{C}$, soit $\theta(U, X)$ l'espace de Fréchet des fonctions analytiques sur $U$ à valeur dans $X$ muni de la topologie de la convergence uniforme sur tous les compacts dans $U$. Suivant $[3,12], T$ satisfait la condition de $\operatorname{Bishop}(\beta)$ si pour tout ouvert $U$ de $\mathbb{C}$,

$$
T_{U}: \theta(U, X) \longrightarrow \theta(U, X), \quad f \longmapsto(z-T) f
$$

est injectif à image fermée. Signalons que la condition de Bishop $(\beta)$ implique la propriété de Dunford $(C)$ qui à son tour implique la SVEP. On dit que $T$ satisfait la propriété de décomposition $(\delta)$ si pour toute paire $\{U, V\}$ d'ouverts recouvrant $\mathbb{C}, X=\mathscr{X}_{T}(\bar{U})+$ $\mathscr{X}_{T}(\bar{V})$, où $\mathscr{X}_{T}(F)=\left\{x: x \in T_{\mathbb{C} \backslash F}(\theta(\mathbb{C} \backslash F, X))\right\}$ pour tout $F$ fermé de $\mathbb{C}$ (voir $[1,12]$ ). D'après [1], les propriétés $(\beta)$ et $(\delta)$ jouent un rôle dual et caractérisent les opérateurs décomposables : $T$ est décomposable si, et seulement si, il satisfait $(\beta)$ et $(\delta)$. Ici, on rappelle que $T$ est décomposable si pour toute paire $\{U, V\}$ d'ouverts recouvrant $\mathbb{C}$, il existe $Y, Z$ deux sous-espaces fermés de $X$ invariants pour $T$ tels que $X=Y+Z, \sigma(T \mid Y) \subseteq U$ et $\sigma(T \mid Z) \subseteq V$. Voir [12] pour plus de détails.

\section{Spectre et spectre local}

La description du spectre d'un shift à poids opérateurs $T$, de poids $\left(T_{n}\right)_{n \geq 0}$ a fait l'objet de plusieurs travaux $[7,8,9,10,11]$. Dans [11], la description du spectre a été faite lorsque les poids $\left(T_{n}\right)_{n \geq 0}$ sont sous la forme $T_{n}=\alpha_{n} A$ où $\left(\alpha_{n}\right)_{n \geq 0}$ est une suite bornée dans $\mathbb{C}$ et $A \in \mathscr{L}(H)$; dans ce cas le spectre est le disque centré en 0 et de rayon $r(T)$. Dans [8], le même résultat est obtenu lorsque les poids $\left(T_{n}\right)_{n \geq 0}$ sont tous inversibles. Dans le cas où les poids $\left(T_{n}\right)_{n \geq 0}$ sont limite d'opérateurs inversibles, ce même résultat fut démontré 
3500 Sur la théorie spectrale locale des shifts à poids opérateurs

dans [9]. Récemment et grâce à des outils de la théorie spectrale locale, nous avons pu donner une description du spectre sans aucune condition sur les poids $\left(T_{n}\right)_{n \geq 0},[10]$.

Théorème 2.1 [10]. Soit T un shift à poids opérateurs quelconques, alors

$$
\sigma(T)=\{\lambda \in \mathbb{C}:|\lambda| \leq r(T)\}
$$

La description du spectre local en un point $x=\left(x_{n}\right)_{n \geq 0}$ tel que les $x_{n}$ sont tous nuls sauf pour un certain $x_{n_{0}}$ est donnée par la proposition suivante.

Proposition 2.2. Soit $T$ un shift à poids opérateurs de poids $\left(T_{n}\right)_{n \geq 0}$ quelconques et soit $a \in H$ non nul. Alors pour tout $x=(0, \cdots 0, a, 0 \ldots), \sigma_{T}(x)$ est stable par symétrie circulaire. En plus,

$$
\sigma_{T}(x)=\left\{\lambda \in \mathbb{C}:|\lambda| \leq r_{T}(x)\right\}
$$

Démonstration. Pour tout $\lambda \in \mathbb{C}$ avec $|\lambda|=1$, on a $T$ et $\lambda T$ sont unitairement équivalents. En effet, $U\left(\left(x_{n}\right)_{n \geq 0}\right)=\left(\lambda^{n} x_{n}\right)_{n \geq 0}$ est unitaire et vérifie $U T U^{*}=\lambda T$. Alors $\sigma_{T}(x)=$ $\sigma_{\lambda T}(U(x))=\sigma_{\lambda T}(x)$. Or $\sigma_{\lambda T}(x)=\lambda \sigma_{T}(x)$, donc $\sigma_{T}(x)=\lambda \sigma_{T}(x)$. Il vient que $\sigma_{T}(x)$ est stable par symétrie circulaire. Comme $\sigma_{T}(x)$ est connexe contenant zéro (voir [10]), il s'en suit que

$$
\sigma_{T}(x)=\left\{\lambda \in \mathbb{C}:|\lambda| \leq r_{T}(x)\right\}
$$

Comme conséquence immédiate de la proposition précédente, on a le corollaire suivant.

Corollaire 2.3. Soit $S_{\alpha}$ un shift à poids unilatéral, alors pour tout $n \geq 0$,

$$
\sigma_{S_{\alpha}}\left(e_{n}\right)=\left\{\lambda \in \mathbb{C}:|\lambda| \leq r_{S_{\alpha}}\left(e_{n}\right)\right\}
$$

Dans toute la suite on s'intéresse au cas où les poids sont tous inversibles. Soit $T$ un shift à poids sur $H^{(\infty)}$, de poids inversibles $\left(T_{n}\right)_{n \geq 0}$. Soit $\pi$ la suite d'éléments de $\mathscr{L}(H)$ définie par

$$
\pi(0)=I, \quad \pi(n)=T_{n-1} T_{n-2} \cdots T_{0} \quad \text { pour } n=1,2, \ldots
$$

Soit $H^{2}(\pi)$ l'espace vectoriel des séries entières formelles à coefficients dans $H, f=$ $\sum_{n=0}^{\infty} \hat{f}(n) z^{n}$ telles que $\|f\|_{\pi}^{2}=\sum_{n=0}^{\infty}\|\pi(n) \hat{f}(n)\|^{2}<\infty$. Alors $\|\cdot\|_{\pi}$ définit une norme sur $H^{2}(\pi)$ qui provient du produit scalaire $\langle f, g\rangle_{\pi}=\sum_{n=0}^{\infty}\langle\pi(n) \hat{f}(n), \pi(n) \hat{g}(n)\rangle$, où $f=$ $\sum_{n=0}^{\infty} \hat{f}(n) z^{n}$ et $g=\sum_{n=0}^{\infty} \hat{g}(n) z^{n}$ sont des éléments de $H^{2}(\pi)$. L'espace $H^{2}(\pi)$ muni de ce produit scalaire est un espace de Hilbert et l'opérateur shift à poids $T$ est unitairement équivalent à $M_{z}$ l'opérateur de multiplication par $z$ sur $H^{2}(\pi)$. En effet, l'opérateur $U$ qui fait correspondre à un élément $f=\sum_{n=0}^{\infty} \hat{f}(n) z^{n}$ de $H^{2}(\pi)$, l'élément $y=(\pi(n) \hat{f}(n))_{n \geq 0}$ de $H^{(\infty)}$, est unitaire et vérifie $U M_{z}=T U$; cf [8]. Dans la suite, il arrive de regarder $T$ comme étant l'opérateur de multiplication $M_{z}$. 
Posons

$$
R_{1}(T)=\lim \inf _{n \rightarrow \infty}\left(\inf _{k \geq 0}\left\|\pi(n+k) \pi(k)^{-1}\right\|\right)^{1 / n} .
$$

ThÉorème 2.4 [8]. Si T un shift à poids inversibles sur $H^{(\infty)}$, alors

(i) $\left\{\lambda \in \mathbb{C}: R_{1}(T) \leq|\lambda| \leq r(T)\right\} \subseteq \sigma_{a p}(T) \subseteq\left\{\lambda \in \mathbb{C}: r_{1}(T) \leq|\lambda| \leq r(T)\right\}$;

(ii) $\quad r(T)=\lim _{n \rightarrow \infty}\left(\sup _{k \geq 0}\left\|\pi(n+k) \pi(k)^{-1}\right\|\right)^{1 / n} \quad$ et $\quad r_{1}(T)=\lim _{n \rightarrow \infty}\left(\inf _{k \geq 0} 1\right.$ / $\left.\left\|\pi(k) \pi(k+n)^{-1}\right\|\right)^{1 / n}$.

\section{SVEP et propriété de Dunford $(C)$}

Il est facile de voir que si $T$ est un shift à poids opérateurs (pas nécessairement inversibles), alors $\sigma_{p}(T) \subseteq\{0\}$, en particulier $T$ vérifie la SVEP.

Soit $T$ un shift à poids opérateurs inversibles. Si $r_{1}(T)>0$, alors clairement l'opérateur $T^{*}$ est surjectif mais non injectif. Par conséquent, il ne satisfait pas la SVEP. La caractérisation de $\sigma_{p}\left(T^{*}\right)$ permet d'avoir mieux.

Soit $r_{2}(T)=1 / \limsup \operatorname{sum}_{n \rightarrow \infty}\left\|\pi(n)^{-1}\right\|^{1 / n}$. Alors pour tout $|\lambda|<r_{2}(T), x \in H$ non nul, on a $\sum_{n=0}^{\infty}\left\|\pi(n)^{*^{-1}} x\right\|^{2}|\lambda|^{2 n}<\infty$ et $\left(\lambda^{n} \pi(n)^{*^{-1}} x\right)_{n \geq 0}$ est dans $N\left(T^{*}-\lambda\right)$.

Dans [8, théorème 5.10], le premier auteur a localisé le spectre ponctuel de $T^{*}$ à l'aide de $R_{2}(T)$ défini par

$$
R_{2}(T)=\sup \left\{|\lambda|: \lambda \in \sigma_{p}\left(T^{*}\right)\right\} .
$$

Théorème $3.1[8]$. Soit $T \in \mathscr{L}\left(H^{(\infty)}\right)$ un shift à poids opérateurs inversibles, alors

$$
\{0\} \cup\left\{\lambda:|\lambda|<R_{2}(T)\right\} \subseteq \sigma_{p}\left(T^{*}\right) \subseteq\left\{\lambda:|\lambda| \leq R_{2}(T)\right\} .
$$

Démonstration. On a par définition de $R_{2}(T):\left\{\lambda:|\lambda| \leq R_{2}(T)\right\} \supseteq \sigma_{p}\left(T^{*}\right)$. Aussi $0 \in$ $\sigma_{p}\left(T^{*}\right)$. Maintenant soit $\lambda \in \sigma_{p}\left(T^{*}\right)$, alors il existe un élément non nul de $H^{(\infty)}, x=$ $\left(x_{n}\right)_{n \geq 0}$ tel que $T^{*} x=\lambda x$. Or $T^{*} x=\left(T_{n}^{*} x_{n+1}\right)_{n \geq 0}$, donc $T^{*} x=\lambda x$ si, et seulement si, $T_{n}^{*} x_{n+1}=\lambda x_{n}$ pour $n=0,1,2, \ldots$ On en déduit donc que pour tout $n \geq 1$, on a $x_{n}=$ $\lambda^{n} \pi(n)^{*-1} x_{0}$. Puisque $x \neq 0$, on doit avoir $x_{0} \neq 0$. Comme $x$ est dans $H^{(\infty)}$, alors

$$
\sum_{n=0}^{\infty}\left\|\pi(n)^{*-1} x_{0}\right\|^{2}|\lambda|^{2 n}<\infty
$$

On voit donc qu'une condition nécessaire et suffisante pour que $\lambda$ soit dans $\sigma_{p}\left(T^{*}\right)$, est qu'il existe un élément $x$ de $H$ tel que

$$
\sum_{n=0}^{\infty}\left\|\pi(n)^{*-1} x\right\|^{2}|\lambda|^{2 n}<\infty, \quad x \neq 0 .
$$

Si donc $\lambda \in \sigma_{p}\left(T^{*}\right)$ et $\mu \in \mathbb{C}$ est tel que $|\mu| \leq|\lambda|$, alors $\mu \in \sigma_{p}\left(T^{*}\right)$. D'où le résultat.

Corollaire 3.2. Sous les même conditions du théorème précédent on a

$$
R_{2}(T)=\sup _{x \in H \backslash\{0\}}\left(\frac{1}{\limsup _{n \rightarrow \infty}\left\|\pi(n)^{*-1}(x)\right\|^{1 / n}}\right) .
$$


3502 Sur la théorie spectrale locale des shifts à poids opérateurs

Démonstration. Nous avons montré que $\lambda \in \sigma_{p}\left(T^{*}\right)$ si, et seulement si, il existe $x$ dans $H$ non nul tel que

$$
\sum_{n=0}^{\infty}\left\|\pi(n)^{*-1} x\right\|^{2}|\lambda|^{2 n}<\infty
$$

Autrement dit,

$$
|\lambda|<\frac{1}{\limsup _{n \rightarrow \infty}\left\|\pi(n)^{*-1}(x)\right\|^{1 / n}} .
$$

Donc

$$
R_{2}(T) \leq \sup _{x \in H \backslash\{0\}}\left(\frac{1}{\limsup _{n \rightarrow \infty}\left\|\pi(n)^{*-1}(x)\right\|^{1 / n}}\right) .
$$

Pour l'autre inégalité, soit $\varepsilon>0$. Alors il existe un élément $y$ dans $H$ non nul tel que

$$
\sup _{x \in H \backslash\{0\}}\left(\frac{1}{\limsup _{n \rightarrow \infty}\left\|\pi(n)^{*-1}(x)\right\|^{1 / n}}\right)-\varepsilon<\frac{1}{\limsup _{n \rightarrow \infty}\left\|\pi(n)^{*-1}(y)\right\|^{1 / n}} .
$$

Donc d'après la preuve du théorème 3.1 on a

$$
\sup _{x \in H \backslash\{0\}}\left(\frac{1}{\limsup _{n \rightarrow \infty}\left\|\pi(n)^{*-1}(x)\right\|^{1 / n}}\right)-\varepsilon \leq R_{2}(T) .
$$

Ce qui achève la démonstration.

La proposition suivante porte une caractérisation de la SVEP de l'adjoint.

Proposition 3.3. Soit $T$ un shift à poids opérateurs inversibles, alors $R_{2}(T)=0$ est une condition nécessaire et suffisante pour que $T^{*}$ satisfasse la SVEP.

Démonstration. Si $R_{2}(T)=0$, alors d'après théorème 3.1 on a $\sigma_{p}\left(T^{*}\right)=\{0\}$ et donc $T^{*}$ satisfait la SVEP. Maintenant si $R_{2}(T)>0$ alors pour $x \in H$ non nul, $f(\mu)=$ $\left(\mu^{n} \pi(n)^{*^{-1}} x\right)_{n \geq 0}$ vérifie $\left(T^{*}-\mu\right) f(\mu)=0$ sur $|\mu|<1 / \limsup _{n \rightarrow \infty}\left\|\pi(n)^{*-1}(x)\right\|^{1 / n}$. Par conséquent, $T^{*}$ ne satisfait pas la SVEP.

Dans le cas où les poids sont des scalaires, on a $R_{2}\left(S_{\alpha}\right)=r_{2}\left(S_{\alpha}\right)$. On retrouve alors le resultat donné par [14].

Corollaire 3.4. Soit $S_{\alpha}$ un shift à poids unilatéral injectif. Alors $S_{\alpha}^{*}$ satisfait la SVEP si, et seulement si, $r_{2}\left(S_{\alpha}\right)=0$.

Exemple 3.5. (1) Soit $A \in \mathscr{L}(H)$ un opérateur inversible et posons $T_{n}=A$ pour tout $n \geq 0$. Alors

$$
r_{2}(T)=\frac{1}{\limsup _{n \rightarrow \infty}\left\|\pi(n)^{-1}\right\|^{1 / n}}=\frac{1}{\limsup _{n \rightarrow \infty}\left\|\left(A^{-1}\right)^{n}\right\|^{1 / n}}=\frac{1}{r\left(A^{-1}\right)} .
$$


Comme $r_{2}(T) \leq R_{2}(T)$, alors $R_{2}(T)>0$ et donc l'adjoint du shift à poids opérateurs $T$, de poids $\left(T_{n}\right)_{n \geq 0}$, ne satisfait pas la SVEP.

(2) Soit $S_{\alpha}$ le shift unilatéral de poids $\left(\alpha_{n}\right)_{n \geq 0}$ avec $\alpha_{0}=1$ et $\alpha_{n}=1+1 / n, \forall n \geq 1$. On vérifie facilement que $r_{2}\left(S_{\alpha}\right)=r\left(S_{\alpha}\right)=1$, donc $S_{\alpha}^{*}$ ne satisfait pas la SVEP.

Remarque 3.6. Soient $A \in \mathscr{L}(H)$ et $\left(\alpha_{n}\right)_{n \geq 0}$ une suite bornée dans $\mathbb{C}$. Soit $T$ le shift à poids, de poids $\left(\alpha_{n} A\right)_{n \geq 0}$. Donc $T=A \otimes S_{\alpha}$. Si $A$ et $S_{\alpha}$ satisfont la condition de Bishop $(\beta)$, alors de [1, théorème 10] il existe $B \in \mathscr{L}\left(K_{1}\right)$ et $R \in \mathscr{L}\left(K_{2}\right)$ décomposables tels que $A=B \mid H$ et $S_{\alpha}=R \mid H$. Il s'ensuit de [2, corollaire 2.5] que $B \otimes R \in \mathscr{L}\left(K_{1} \otimes K_{2}\right)$ est décomposable, en particulier satisfait la condition de Bishop $(\beta)$. Comme la condition de Bishop $(\beta)$ est stable par restriction, alors $T=B \otimes R \mid H \otimes H$ satisfait la condition de Bishop $(\beta)$ en particulier la propriété de Dunford $(C)$.

La propriété de Dunford $(C)$ joue un rôle important dans la théorie spectrale locale ([12]) et dans d'autres domaines de la théorie des opérateurs (voir par exemple [6]).

Dans le cas où les poids sont inversibles, le théorème suivant, qui généralise le résultat de [13], donne une condition suffisante qui assure la propriété de Dunford $(C)$. Le résultat est obtenu aussi dans [12, proposition 1.6.5] lorsque $r_{1}(T)=r(T)$. Ici le théorème suivant améliore le résultat de [12, proposition 1.6.5]. Dans la preuve on regarde $T$ comme étant l'opérateur $M_{z}$ sur $H^{2}(\pi)$.

ThÉorème 3.7. Soit $T$ un shift à poids inversibles de poids $\left(T_{n}\right)_{n \geq 0}$. Si $r_{2}(T)=r(T)$, alors pour tout $x \in H^{(\infty)}$ non nul, $\sigma_{T}(x)=\sigma(T)$. En particulier, $T$ satisfait la propriété de Dunford (C).

Démonstration. Sans perte de généralité supposons que $r_{2}(T)=r(T)>0$. Soit $x \in H$ fixé. Pour $|\lambda|<r_{2}(T)$, on a $h_{x, \lambda}=\sum_{n=0}^{\infty}\left(\bar{\lambda}^{n} \pi(n)^{-1} \pi(n)^{*^{-1}} x\right) z^{n}$ est dans $N(T-\lambda)^{*}$. Si $f \in H^{2}(\pi)$, alors

$$
\left\langle f, h_{x, \lambda}\right\rangle_{\pi}=\sum_{n=0}^{\infty}\left\langle\pi(n) \hat{f}(n), \bar{\lambda}^{n} \pi(n)^{*^{-1}} x\right\rangle=\sum_{n=0}^{\infty}\langle\hat{f}(n), x\rangle \lambda^{n} .
$$

Donc pour chaque $f \in H^{2}(\pi)$, la fonction $\lambda \rightarrow \sum_{n=0}^{\infty}\langle\hat{f}(n), x\rangle \lambda^{n}$ est analytique sur $\{\lambda \in$ $\left.\mathbb{C}:|\lambda|<r_{2}(T)\right\}$.

S'il existe un $f \in H^{2}(\pi)$ non nul avec $\sigma_{T}(f)$ inclus strictement dans $\sigma(T)$, il existera alors un ouvert $U$ inclus dans $\left\{\lambda \in \mathbb{C}:|\lambda|<r_{2}(T)\right\}$ et $\varphi: U \rightarrow H^{2}(\pi)$ analytique avec $(T-\lambda) \varphi(\lambda)=f, \forall \lambda \in U$. Soit $x \in H$ fixé et soit $\lambda \in U$, on a

$$
\sum_{n=0}^{\infty}\langle\hat{f}(n), x\rangle \lambda^{n}=\left\langle f, h_{x, \lambda}\right\rangle_{\pi}=\left\langle(T-\lambda) \varphi(\lambda), h_{x, \lambda}\right\rangle_{\pi}=\left\langle\varphi(\lambda),(T-\lambda)^{*} h_{x, \lambda}\right\rangle_{\pi}=0
$$

Donc pour tout $\lambda \in U, \sum_{n=0}^{\infty}\langle\hat{f}(n), x\rangle \lambda^{n}=0$. Et comme elle est analytique sur $\{\mu \in \mathbb{C}$ : $\left.|\mu|<r_{2}(T)\right\}$, alors elle est nulle. D'où, $\langle\hat{f}(n), x\rangle=0, \forall n \geq 0$. Et ceci pour tout $x \in H$. Par conséquent, $\hat{f}(n)=0, \forall n \geq 0$, ce qui entraîne $f \equiv 0$. D'où la contradiction. Donc pour tout $f \in H^{2}(\pi)$ non nul,

$$
\sigma_{T}(f)=\sigma(T)
$$


Maintenant soit $F$ une partie fermée de $\mathbb{C}$. Alors d'après l'égalité précédente, on a $H_{T}^{(\infty)}(F)=H^{(\infty)}$ si $F$ contient $\sigma(T)$, et $H_{T}^{(\infty)}(F)=\{0\}$ dans le cas contraire. Donc $T$ satisfait la propriété de Dunford $(C)$.

Exemple 3.8. (1) Soit $\left(T_{n}\right)_{n \geq 0}$ une suite d'opérateurs unitaires, alors le shift à poids $T$ de poids $\left(T_{n}\right)_{n \geq 0}$ vérifie $r_{2}(T)=r(T)=1$. Donc $T$ satisfait la propriété de Dunford $(C)$.

(2) Soit $A \in \mathscr{L}(H)$ un opérateur inversible tel que $\sigma(A) \subset\{\lambda \in \mathbb{C}:|\lambda|=1\}$. Soit $\left(\alpha_{n}\right)_{n \geq 0}$ une suite bornée de nombres réels positifs vérifiant

$$
\lim \inf _{n \rightarrow \infty}\left(\alpha_{0} \cdots \alpha_{n}\right)^{1 / n}=\sup _{n \geq 0} \alpha_{n}
$$

alors le shift à poids $T$, de poids $\left(\alpha_{n} A\right)_{n \geq 0}$, vérifie $r_{2}(T)=r(T)$. Donc $T$ satisfait la propriété de Dunford $(C)$ d'après le théorème 3.7.

Dans la proposition suivante, nous donnerons une condition nécessaire pour qu'un shift à poids unilatéral injectif satisfasse la propriété de Dunford $(C)$. Ce résultat est obtenu aussi par $[4,14]$, mais ici on donne une démonstration directe.

Proposition 3.9. Soit $S_{\alpha}$ un shift à poids unilatéral injectif satisfaisant la propriété de Dunford $(C)$. Alors pour tout $x \in H$ non nul, $r_{S_{\alpha}}(x)=r\left(S_{\alpha}\right)$.

Démonstration. Montrons d'abord que $r_{S_{\alpha}}\left(e_{n}\right)=r\left(S_{\alpha}\right)$ pour tout $n \geq 0$. Supposons l'existence d'un $n_{0}$ tel que $r_{S_{\alpha}}\left(e_{n_{0}}\right)=\lim _{p \rightarrow \infty} \sup \left(\alpha_{n_{0}} \alpha_{n_{0}+1} \cdots \alpha_{n_{0}+p-1}\right)^{1 / p}<r\left(S_{\alpha}\right)$. Soient $D$ le disque fermé de centre 0 et de rayon $r_{S_{\alpha}}\left(e_{n_{0}}\right)$ et $M=\max \left\{\left\|S_{\alpha}\right\|, 1\right\}$.

Pour $0 \leq n<n_{0}$, on a $\left\|S_{\alpha}^{p} e_{n}\right\|=\left(\alpha_{n} \cdots \alpha_{n_{0}-1}\right)\left(\alpha_{n_{0}} \cdots \alpha_{n+p-1}\right)$. Par suite,

$$
\begin{aligned}
\left\|S_{\alpha}^{p} e_{n}\right\|^{1 / p} & =\left(\alpha_{n} \cdots \alpha_{n_{0}-1}\right)^{1 / p}\left(\left(\alpha_{n_{0}} \cdots \alpha_{n_{0}+\left(n-n_{0}+p\right)-1}\right)^{1 /\left(n-n_{0}+p\right)}\right)^{\left(n-n_{0}+p\right) / p} \\
& \leq M^{\left(n_{0}-n\right) / p}\left(\left(\alpha_{n_{0}} \cdots \alpha_{n_{0}+\left(n-n_{0}+p\right)-1}\right)^{1 /\left(n-n_{0}+p\right)}\right)^{\left(n-n_{0}+p\right) / p} .
\end{aligned}
$$

Donc $r_{S_{\alpha}}\left(e_{n}\right) \leq r_{S_{\alpha}}\left(e_{n_{0}}\right)$ et par conséquent $e_{n} \in H_{S_{\alpha}}(D)$ pour $0 \leq n<n_{0}$.

Soit $n>n_{0}$. On a $S_{\alpha}^{n-n_{0}} e_{n_{0}}=\left(\alpha_{n_{0}} \cdots \alpha_{n-1}\right) e_{n}$. Comme $e_{n_{0}}$ est dans $H_{S_{\alpha}}(D)$ qui est un sous espace invariant pour $S_{\alpha}$, alors $e_{n} \in H_{S_{\alpha}}(D)$.

Par conséquent, $e_{n} \in H_{S_{\alpha}}(D), \forall n \geq 0$. Puisque $S_{\alpha}$ satisfait la propriété de Dunford $(C)$, alors $H_{S_{\alpha}}(D)$ est fermé et donc $H=H_{S_{\alpha}}(D)$. Donc $\sigma\left(S_{\alpha}\right) \subseteq D$. Et par suite, $r\left(S_{\alpha}\right) \leq$ $r_{S_{\alpha}}\left(e_{n_{0}}\right)<r\left(S_{\alpha}\right)$, d'où la contradiction. Alors pour tout $n \geq 0, r_{S_{\alpha}}\left(e_{n}\right)=r\left(S_{\alpha}\right)$.

Maintenant, soit $x \in H$ non nul. Si $x=\sum_{n=0}^{\infty} x_{n} e_{n}$, il existe donc un $n_{0}$ tel que $x_{n_{0}} \neq 0$. On a

$$
\left\|S_{\alpha}^{p} x\right\|^{2}=\sum_{n=0}^{\infty}\left|x_{n}\right|^{2}\left\|S_{\alpha}^{p} e_{n}\right\|^{2} \geq\left|x_{n_{0}}\right|^{2}\left\|S_{\alpha}^{p} e_{n_{0}}\right\|^{2} .
$$

En conséquence, $r_{S_{\alpha}}(x)=\lim _{p \rightarrow \infty} \sup \left\|S_{\alpha}^{p} x\right\|^{1 / p} \geq r_{S_{\alpha}}\left(e_{n_{0}}\right)=r\left(S_{\alpha}\right)$.

Corollaire 3.10. Soit $S_{\alpha}$ un shift à poids unilatéral injectif satisfaisant la propriété de Dunford $(C)$. Alors $\sigma_{S_{\alpha}}\left(e_{n}\right)=\sigma\left(S_{\alpha}\right)$, pour tout $n \geq 0$.

Démonstration. Il suffit d'appliquer la proposition 3.9 et le corollaire 2.3. 
Dans le cas d'un shift à poids unilatéral injectif à poids scalaires, nous avons montré que le fait que $r_{S_{\alpha}}(x)=r\left(S_{\alpha}\right)$ pour tout $x$ non nul est une condition nécessaire pour que l'opérateur shift satisfasse la propriété de Dunford $(C)$. Comme le montre l'exemple suivant, ce résultat n'est plus vrai dans le cas d'un shift à poids opérateurs inversibles.

Exemple 3.11. Soit $A$ l'opérateur défini par $A e_{0}=(1 / 2) e_{0}$ et $A e_{n}=e_{n}, \forall n \geq 1$, alors $A$ est inversible. Considérons le shift à poids opérateurs $T$ de poids $\left(T_{n}\right)_{n \geq 0}$ avec $T_{n}=A$, $\forall n \geq 0$. Soit $x=\left(e_{0}, 0,0, \ldots\right)$ et $y=\left(e_{1}, 0,0, \ldots\right)$, alors

$$
\left\|T^{p} x\right\|=\left\|A^{p} e_{0}\right\|, \quad\left\|T^{p} y\right\|=\left\|A^{p} e_{1}\right\| .
$$

Donc

$$
r_{T}(x)=r_{T_{0}}\left(e_{0}\right)=\frac{1}{2}<1=r_{T_{0}}\left(e_{1}\right)=r_{T}(y),
$$

tandis que $T$ satisfait la propriété de Dunford $(C)$. En effet, soit $S$ le shift unilateral sans poids, alors $A$ et $S$ sont en particulier hyponormaux. Alors d'après [5], $A$ et $S$ satisfont la condition de Bishop $(\beta)$. Il vient de la remarque 3.6 que $A \otimes S=T$ satisfait la condition de Bishop $(\beta)$ et par suite la propriété de Dunford $(C)$.

\section{Condition de Bishop $(\beta)$ et décomposabilité}

Plusieurs études ont été faites pour caractériser les classes d'opérateurs ayant la condition de Bishop $(\beta)$. A titre d'exemple, les opérateurs hyponormaux et d'une manière générale, les $p$-hyponormaux satisfont la condition de Bishop $(\beta)$ (voir [5]). Toutefois, une caractérisation complète (ou satisfaisante) de la condition de Bishop $(\beta)$ des shifts à poids reste inachevée. Des conditions suffisantes ont été données dans $[13,14]$. Dans cette section nous donnerons une condition qui assure la condition de Bishop $(\beta)$ pour un shift à poids opérateurs inversibles. Nous montrons aussi qu'un shift à poids opérateurs est décomposable si, et seulement si, il est quasinilpotent.

Lemme 4.1. Soit $T$ un shift à poids opérateurs inversibles tel que $r_{2}(T)>0$. Soit $f \in H^{2}(\pi)$ avec $f(z)=\sum_{n=0}^{\infty} \hat{f}(n) z^{n}$. Alors pour tout $|\lambda|<r_{2}(T), f(\lambda)=\sum_{n=0}^{\infty} \hat{f}(n) \lambda^{n}$ est bien définie.

Démonstration. Soit $\psi: H \rightarrow \mathbb{C}$ définie par $\psi(x)=\sum_{n=0}^{\infty}\langle\hat{f}(n), x\rangle \lambda^{n}$. Donc $\psi(x)=$ $\left\langle f, h_{x, \lambda}\right\rangle_{\pi}$. Par suite, $\psi$ est linéaire car $h_{x+\mu y, \lambda}=h_{x, \lambda}+\mu h_{y, \lambda}$. De plus

$$
|\psi(x)|=\left|\left\langle f, h_{x, \lambda}\right\rangle_{\pi}\right| \leq\|f\|_{\pi}\left(\sum_{n=0}^{\infty}\left\|\pi(n)^{*-1}|\lambda|^{n}\right\|^{2}\right)^{1 / 2}\|x\| .
$$

Donc $\psi$ est continue. Par application du théorème de représentation de Riesz, il existe un unique $f(\lambda) \in H$ tel que

$$
\langle f(\lambda), x\rangle=\psi(x)=\left\langle f, h_{x, \lambda}\right\rangle_{\pi}
$$

Soit $S_{n}(z)=\sum_{k=0}^{n} \hat{f}(k) z^{k}$. On a

$$
\left|\left\langle f(\lambda)-S_{n}(\lambda), x\right\rangle\right|=\left|\left\langle f-S_{n}, h_{x, \lambda}\right\rangle_{\pi}\right| \leq M\left\|f-S_{n}\right\|_{\pi}\|x\|,
$$


où $M$ une constante. Donc $\left\|f(\lambda)-S_{n}(\lambda)\right\| \leq M\left\|f-S_{n}\right\|_{\pi}$. Par conséquent, $S_{n}(\lambda)$ converge vers $f(\lambda)$. D'où $f(\lambda)=\sum_{n=0}^{\infty} \hat{f}(n) \lambda^{n}$.

Théorème 4.2. Soit $T$ un shift à poids inversibles de poids $\left(T_{n}\right)_{n \geq 0}$. Si $R_{1}(T)<r_{2}(T)$, alors $T$ ne satisfait pas la condition de Bishop $(\beta)$.

Démonstration. Soit $D$ le disque ouvert de centre 0 et de rayon $r_{2}(T)$. Pour $\lambda \in D$, soit $H_{\lambda}=\left\{f \in H^{2}(\pi): f(\lambda)=0\right\}$. Alors l'ensemble des polynômes dans $H_{\lambda}$ est dense dans $H_{\lambda}$. En effet, si $f \in H^{2}(\pi)$ est tel que $f(\lambda)=0$, il vient du lemme 4.1 que

$$
f=\hat{f}(0)+\sum_{n=1}^{\infty} \hat{f}(n) z^{n}=-\sum_{n=1}^{\infty} \hat{f}(n) \lambda^{n}+\sum_{n=1}^{\infty} \hat{f}(n) z^{n} .
$$

Si $q_{n}=-\sum_{k=1}^{n} \hat{f}(k) \lambda^{k}+\sum_{k=1}^{n} \hat{f}(k) z^{k}$, alors $q_{n}(\lambda)=0$, en plus $q_{n}$ converge vers $f$ dans $H^{2}(\pi)$. Il s'ensuit donc que

$$
\left\{p \in H^{2}(\pi) \text {, polynôme }: p(\lambda)=0\right\} \subseteq(T-\lambda) H^{2}(\pi) \subseteq H_{\lambda} \text {. }
$$

Maintenant pour montrer que $T$ ne satisfait pas la condition de Bishop $(\beta)$, il suffit de montrer que $T_{D}$ n'est pas à image fermée.

Si $(T-\lambda) H^{2}(\pi)=H_{\lambda}$, donc $(T-\lambda) H^{2}(\pi)$ est fermé et par suite $\lambda \notin \sigma_{a p}(T)$. Alors d'après théorème 2.4 on a $|\lambda|<R_{1}(T)$. Soit donc $\lambda_{0}$ avec $R_{1}(T) \leq\left|\lambda_{0}\right|<r_{2}(T)$ et soit $f \in$ $H_{\lambda_{0}} \backslash\left(T-\lambda_{0}\right) H^{2}(\pi)$. Soit $\left(P_{n}\right)_{n \geq 0}$ la suite des polynômes dans $H_{\lambda_{0}}$ qui converge vers $f$. Posons $\varphi(\lambda)=f(z)-f(\lambda)$ et $\varphi_{n}(\lambda)=P_{n}(z)-P_{n}(\lambda), \forall n \geq 0$. Comme $f(z)=\varphi\left(\lambda_{0}\right)$, alors il n'existe pas de fonction $h$, analytique sur $D$ et à valeur dans $H^{2}(\pi)$, vérifiant $\varphi=(T-$ $\mu) h(\mu)$. Si elle existe on aura $f=\varphi\left(\lambda_{0}\right)=\left(T-\lambda_{0}\right) h\left(\lambda_{0}\right)$ et par suite $f \in\left(T-\lambda_{0}\right) H^{2}(\pi)$. D'autre part, pour tout $\lambda \in D$ et pour tout $n \geq 0$, il existe $q_{n}(\lambda)$ polynôme en $z$ analytique en $\lambda$ avec $\varphi_{n}(\lambda)=(z-\lambda) q_{n}(\lambda)=(T-\lambda) q_{n}(\lambda)$.

Il reste à montrer que $\varphi_{n}$ converge vers $\varphi$ dans $\theta\left(D, H^{2}(\pi)\right)$. Soit $r<r_{2}(T)$, on a

$$
\begin{aligned}
\left\|\varphi(\lambda)-\varphi_{n}(\lambda)\right\|_{\pi} & =\left\|f(z)-P_{n}(z)+P_{n}(\lambda)-f(\lambda)\right\|_{\pi} \\
& \leq\left\|f-P_{n}\right\|_{\pi}+\left\|P_{n}(\lambda)-f(\lambda)\right\| \\
& \leq\left\|f-P_{n}\right\|_{\pi}+\sup _{\|x\|=1}\left|\left\langle P_{n}(\lambda)-f(\lambda), x\right\rangle\right| \\
& =\left\|f-P_{n}\right\|_{\pi}+\sup _{\|x\|=1}\left|\left\langle P_{n}-f, h_{x, \lambda}\right\rangle_{\pi}\right| \\
& =\left\|f-P_{n}\right\|_{\pi}\left(1+\sup _{\|x\|=1}\left\|h_{x, \lambda}\right\|\right) \\
& =\left\|f-P_{n}\right\|_{\pi}\left(1+\left(\sum_{k=0}^{\infty}\left\|\pi(k)^{*^{-1}}\right\|^{2} r^{2 k}\right)^{1 / 2}\right)
\end{aligned}
$$

Par conséquent, $T$ ne satisfait pas la condition de Bishop $(\beta)$.

Dans le cas d'un shift à poids scalaires injectif, $R_{1}\left(S_{\alpha}\right)=r_{1}\left(S_{\alpha}\right)$. On obtient alors le résultat donné dans [13]. 
L'opérateur dans l'exemple suivant satisfait la propriété de Dunford $(C)$ sans satisfaire la condition de Bishop $(\beta)$.

Exemple 4.3. Soit $T_{0}$ un opérateur inversible sur $H$ avec $\left\|T_{0}\right\|=1$. Soit $T_{1}=r I$ tel que $0<r<1$. Posons

$$
T_{2 n+1}=T_{1}, \quad T_{2 n}=T_{1}^{-1} \quad \text { pour } n \geq 1
$$

Soit $T$ le shift à poids, de poids $\left(T_{n}\right)_{n \geq 0}$. On vérifie facilement que

$$
\pi(2 n)=T_{1} T_{0}, \quad \pi(2 n+1)=T_{0}
$$

Alors $R_{1}(T)=0<1=r_{2}(T)=r(T)$. Il s'ensuit du théorèmes 3.7 et 4.2 que $T$ satisfait la propriété de Dunford $(C)$ sans satisfaire la condition de Bishop $(\beta)$.

Une condition suffisante qui assure la condition de Bishop $(\beta)$ est donnée par le théorème suivant.

ThÉorème 4.4. Soit $S_{\alpha}$ un shift à poids unilatéral injectif de poids $\left(\alpha_{n}\right)_{n \geq 0}$ tel que $r_{1}\left(S_{\alpha}\right)=$ $r\left(S_{\alpha}\right)=1$. Soit

$$
\varphi_{1, n}\left(S_{\alpha}\right)=\sup \left\{\left\|S_{\alpha}^{k}\right\|^{1 / n}-1, k=1, \ldots, n\right\}
$$

et

$$
\varphi_{2, n}\left(S_{\alpha}\right)=\sup \left\{\frac{1}{m\left(S_{\alpha}^{k}\right)^{1 / n}}-1, k=1, \ldots, n\right\} .
$$

Si $\varphi_{n}\left(S_{\alpha}\right)=\sup \left\{\varphi_{1, n}\left(S_{\alpha}\right), \varphi_{2, n}\left(S_{\alpha}\right)\right\}$ vérifie $\sum_{n=1}^{\infty}\left(\varphi_{n}\left(S_{\alpha}\right)\right) / n<\infty$, alors $S_{\alpha}$ satisfait la condition de Bishop $(\beta)$.

Démonstration. Soit $\alpha_{-n}=1$ pour $n=1,2 \ldots$ Soit $R e_{n}=\alpha_{n} e_{n+1}, \forall n \in \mathbb{Z}$. Alors $R$ est un shift à poids bilatéral injectif et $R \mid l^{2}(\mathbb{C})=S_{\alpha}$. Calculons d'abord, $r_{1}^{+}(R), r^{+}(R), r_{1}^{-}(R)$ et $r^{-}(R)$. On a

$$
\begin{aligned}
r_{1}^{+}(R) & =\lim _{n \rightarrow \infty}\left(\inf _{j \geq 0} \frac{\beta(n+j)}{\beta(j)}\right)^{1 / n}=\lim _{n \rightarrow \infty}\left(\inf _{j \geq 0} \alpha_{j} \cdots \alpha_{n+j-1}\right)^{1 / n} \\
& =\lim _{n \rightarrow \infty}\left(m\left(S_{\alpha}^{n}\right)\right)^{1 / n}=r_{1}\left(S_{\alpha}\right)=r\left(S_{\alpha}\right)=1 .
\end{aligned}
$$

Et $r^{+}(R)=\lim _{n \rightarrow+\infty}\left(\sup _{j \geq 0}(\beta(n+j) / \beta(j))\right)^{1 / n}=r\left(S_{\alpha}\right)=1$, donc $r_{1}^{+}(R)=r^{+}(R)=1$. Aussi $r_{1}^{-}(R)=\lim _{n \rightarrow+\infty}\left(\inf _{j<0}(\beta(-n+j) / \beta(j))\right)^{1 / n}=1$ et $r^{-}(R)=1$. Donc, d'après [15], $r(R)=$ $r_{1}(R)=1$. 
3508 Sur la théorie spectrale locale des shifts à poids opérateurs

Maintenant, posons $\varepsilon_{n}=\left\|R^{n}\right\|^{1 / n}-1, \eta_{n}=\left\|R^{-n}\right\|^{1 / n}-1$ et $\Psi_{n}=\sup \left\{\varepsilon_{n}, \eta_{n}\right\}$. On a

$$
\begin{aligned}
\left\|R^{n}\right\| & =\sup _{k} \alpha_{k} \cdots \alpha_{k+n-1} \\
& =\sup \left\{\sup _{k \geq 0} \alpha_{k} \cdots \alpha_{k+n-1}, \sup _{k<1-n} \alpha_{k} \cdots \alpha_{k+n-1}, \sup _{1-n \leq k<0} \alpha_{k} \cdots \alpha_{k+n-1}\right\} \\
& =\sup \left\{\left\|S^{n}\right\|, 1, \sup _{1-n \leq k<0} \alpha_{k} \cdots \alpha_{k+n-1}\right\} .
\end{aligned}
$$

Par suite, $\varepsilon_{n} \leq \sup \left\{\left\|S_{\alpha}^{k}\right\|^{1 / n}-1, k=1, \ldots, n\right\}$. Et

$$
\begin{aligned}
\left\|R^{-n}\right\| & =\sup _{j} \frac{\beta(j)}{\beta(n+j)} \\
& =\sup \left\{\sup _{j \geq 0} \frac{\beta(j)}{\beta(n+j)}, \sup _{j \leq-n} \frac{\beta(j)}{\beta(j+n)}, \sup _{-n<j<0} \frac{\beta(j)}{\beta(j+n)}\right\} \\
& \leq \sup \left\{\frac{1}{m\left(S_{\alpha}^{n}\right)}, 1, \frac{1}{m\left(S_{\alpha}\right)}, \ldots, \frac{1}{m\left(S_{\alpha}^{n-1}\right)}\right\} .
\end{aligned}
$$

Donc $\eta_{n} \leq \sup \left\{\left(1 / m\left(S_{\alpha}^{k}\right)^{1 / n}\right)-1, k=1, \ldots, n\right\}$. Par conséquent, $\sum_{n=1}^{\infty}\left(\Psi_{n} / n\right)<\infty$ et donc d'après [17, théorème 2$], R$ est décomposable. D'où, d'après [1, théorème 10$], S_{\alpha}$ satisfait la condition de Bishop $(\beta)$.

La décomposabilité d'un shift à poids opérateurs quelconques est équivalente à la quasinilpotence.

Théorème 4.5. Soit T un shift à poids opérateurs quelconques. Alors les propriétés suivantes sont équivalentes :

(i) T est décomposable;

(ii) T satisfait la propriété $(\delta)$;

(iii) $T$ est quasinilpotent.

Démonstration. Comme $\bigcap_{n \geq 1} T^{n}\left(H^{(\infty)}\right)=\{0\}$, alors le résultat se déduit du théorème 1.6.3 de [12].

\section{Remerciements}

Une grande partie de ce travail fait partie de la thèse de D. E. S. du second auteur [18]. Les auteurs tiennent à remercier les Professeurs M. Boumazgour et E. H. Zerouali pour leurs remarques lors de la rédaction de ce travail. Ils remercient aussi le référé pour ses suggestions fructueuses.

\section{Le bibliographie}

[1] E. Albrecht and J. Eschmeier, Analytic functional models and local spectral theory, Proc. London Math. Soc. (3) 75 (1997), no. 2, 323-348. 
[2] E. Albrecht, J. Eschmeier, and M. M. Neumann, Some topics in the theory of decomposable operators, Advances in Invariant Subspaces and Other Results of Operator Theory (Timişoara and Herculane, 1984), Oper. Theory Adv. Appl., vol. 17, Birkhäuser, Basel, 1986, pp. 15-34.

[3] E. Bishop, A duality theorem for an arbitrary operator, Pacific J. Math. 9 (1959), 379-397.

[4] A. Bourhim, On the local spectral properties of weighted shift operators, Studia Math. 163 (2004), no. 1, 41-69.

[5] B. P. Duggal, p-hyponormal operators satisfy Bishop's condition ( $\beta$ ), Integral Equations Operator Theory 40 (2001), no. 4, 436-440.

[6] B. P. Duggal and S. V. Djordjević, Dunford's property $(C)$ and Weyl's theorems, Integral Equations Operator Theory 43 (2002), no. 3, 290-297.

[7] N. Faour and R. Khalil, On the spectrum of weighted operator shifts, Houston J. Math. 13 (1987), no. 4, 549-556.

[8] M. Houimdi, Propriétés spectrales des opérateurs shifts, modèles de similitudes et phénomènes d'explosion du spectre, thèse de 3ème cycle, Université Bordeaux 1, Talence, 1984.

[9] M. Houimdi and M. Ouali, Sur le spectre d'un shift à poids opérateurs [On the spectrum of an operator-weighted shift], Extracta Math. 13 (1998), no. 3, 327-333 (French).

[10] M. Houimdi and H. Zguitti, Théorie spectrale locale appliquée aux opérateurs shifts [Local spectral theory applied to shift operators], Extracta Math. 14 (1999), no. 3, 329-332 (French).

[11] P. N. Knyazev, The spectrum of a unilateral shift with operator weights that are scalar-multiple to a fixed operator, Dokl. Akad. Nauk BSSR 29 (1985), no. 4, 304-305, 380.

[12] K. B. Laursen and M. M. Neumann, An Introduction to Local Spectral Theory, London Mathematical Society Monographs. New Series, vol. 20, Clarendon Press, Oxford University Press, New York, 2000.

[13] T. L. Miller and V. G. Miller, An operator satisfying Dunford's condition (C) but without Bishop's property $(\beta)$, Glasgow Math. J. 40 (1998), no. 3, 427-430.

[14] T. L. Miller, V. G. Miller, and M. M. Neumann, Local spectral properties of weighted shifts, J. Operator Theory 51 (2004), no. 1, 71-88.

[15] A. L. Shields, Weighted shift operators and analytic function theory, Topics in Operator Theory, Math. Surveys, no. 13, American Mathematical Society, Rhode Island, 1974, pp. 49-128.

[16] S. L. Sun, Decomposability of weighted shift operators and hyponormal operators on Hilbert spaces, Chinese Ann. Math. Ser. A 5 (1984), no. 5, 575-584 (Chinese).

[17] Spectral decomposition of weighted shift operators, Acta Math. Sinica (N.S.) 2 (1986), no. 4, 367-376.

[18] H. Zguitti, Théorie spectrale locale appliquée aux différentes classes d'opérateurs, thèse de 3ème cycle, Université Cadi Ayyad, Faculté des Sciences Semlalia, Marrakech, 1998.

Mohamed Houimdi : Département de Mathématiques, Faculté des Sciences Semlalia, Université Cadi Ayyad, BP 2390, Marrakech, Maroc

Courrier électronique: houimdi@ucam.ac.ma

Hassane Zguitti : Département de Mathématiques et Informatique, Faculté des Sciences de Rabat, Université Mohammed V Agdal, BP 1014, Agdal, Rabat, Maroc

Courrier électronique: zguitti@hotmail.com 


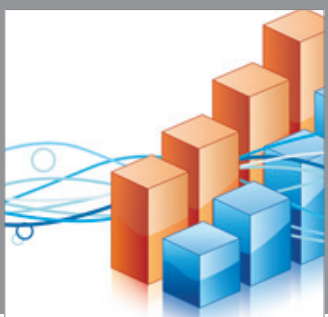

Advances in

Operations Research

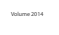

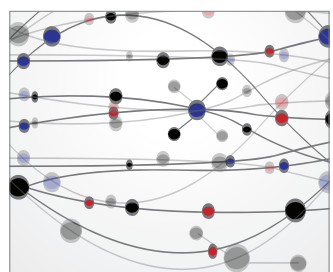

\section{The Scientific} World Journal
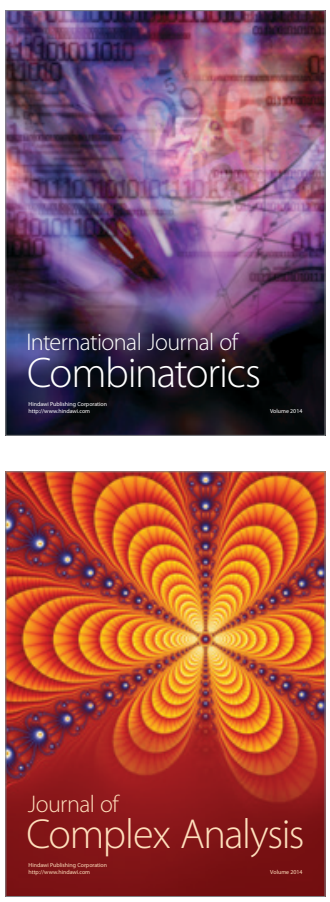

International Journal of

Mathematics and

Mathematical

Sciences
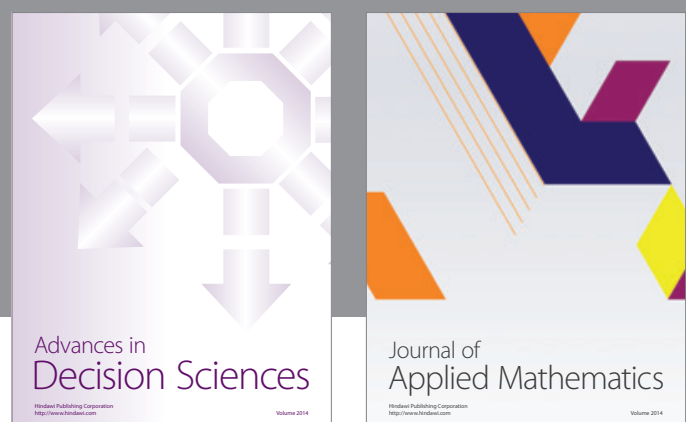

Journal of

Applied Mathematics
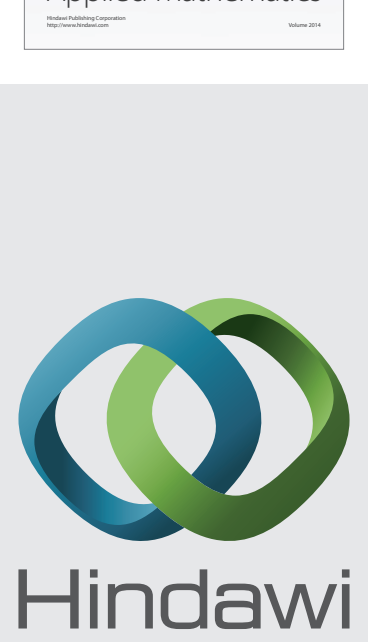

Submit your manuscripts at http://www.hindawi.com
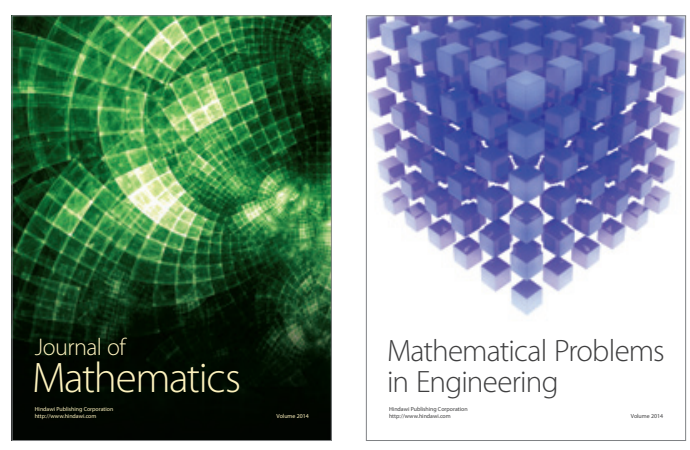

Mathematical Problems in Engineering
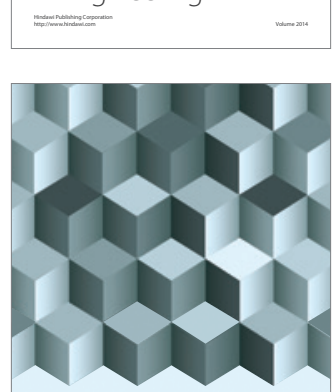

Journal of

Function Spaces
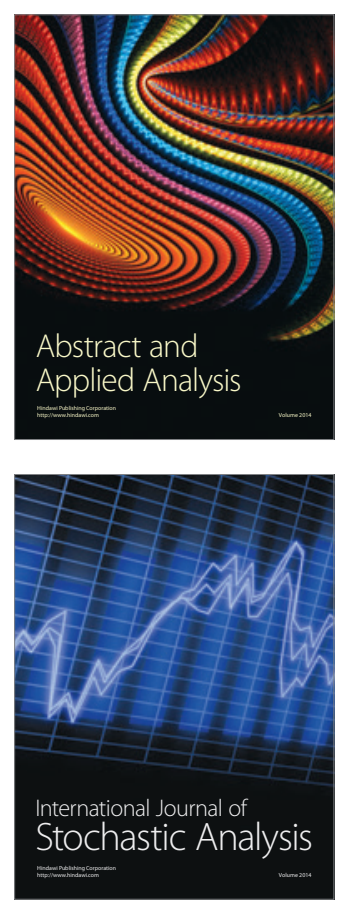

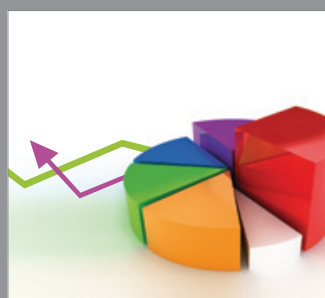

ournal of

Probability and Statistics

Promensencen
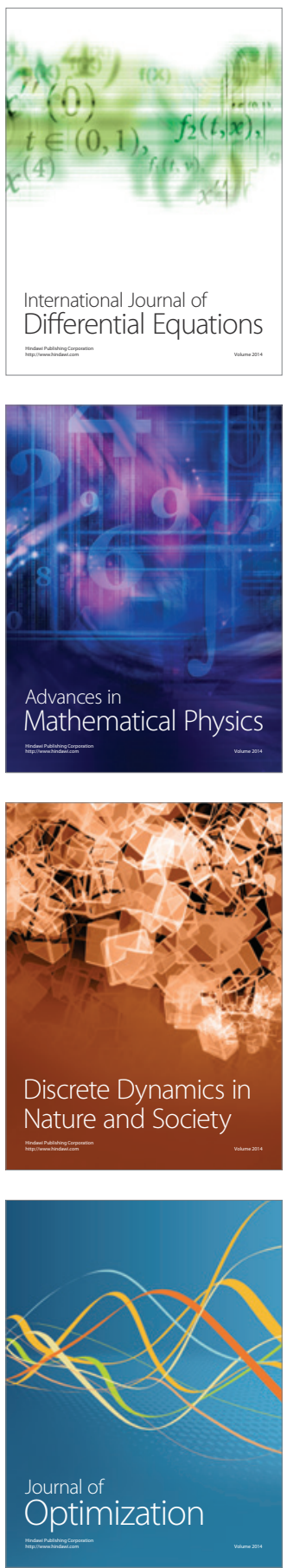